\title{
Stimulation of growth on a deficient medium of a Klebsiella aerogenes species by sulphonamide, para-amino benzoic acid, nitrofurantoin, and some other substances
}

\author{
D. McGHiE, J. G. P. HUTChisON, AND R. G. FINCH
}

From the Regional Public Health Laboratory, East Birmingham Hospital, Birmingham

SYNOPSIS A strain of Klebsiella aerogenes is described which was unable to grow from light inocula on $5 \%$ lysed horse blood diagnostic sensitivity test (DST) agar. It was able to grow on DST agar without added horse cells and MacConkey and nutrient agar. Growth on a deficient medium was promoted by sulphonamides and nitrofurantoin as well as by para-amino benzoic acid, paraamino salicylic acid, procaine hydrochloride, thymine, and uracil.

The organism, Klebsiella aerogenes, was first isolated from a urine specimen on MacConkey agar (Oxoid). When antimicrobial sensitivity testing was attempted using DST agar (Oxoid) enriched with 5\% lysed horse blood and a Mastring (Mast Laboratories), bacterial growth took place as shown in Figure 1 . The centre of the plate was free from growth and what appeared to be stimulated growth was seen associated with the sulphathiazole (no. 8) and nitrofurantoin (no. 5) positions. There follows an account of some features of this organism.

\section{Materials and Methods}

\section{MATERIALS}

Diagnostic sensitivity test (Oxoid) agar with $5 \%$ saponin lysed horse blood was used throughout to test the effect of growth-promoting substances. Other media such as MacConkey agar (Oxoid) or $1 \%$ peptone water (Oxoid) were not critical. The organism, Klebsiella aerogenes, was isolated from the urine of a male inpatient aged 55 who was a chronic bronchitic with asthma, from whom urine was taken as a routine (no clinical evidence of infection). We have no evidence of co-trimoxazole treatment at any time, but in retrospect the possibility of cross infection from the patients on the same ward, treated with co-trimoxazole cannot be discounted.

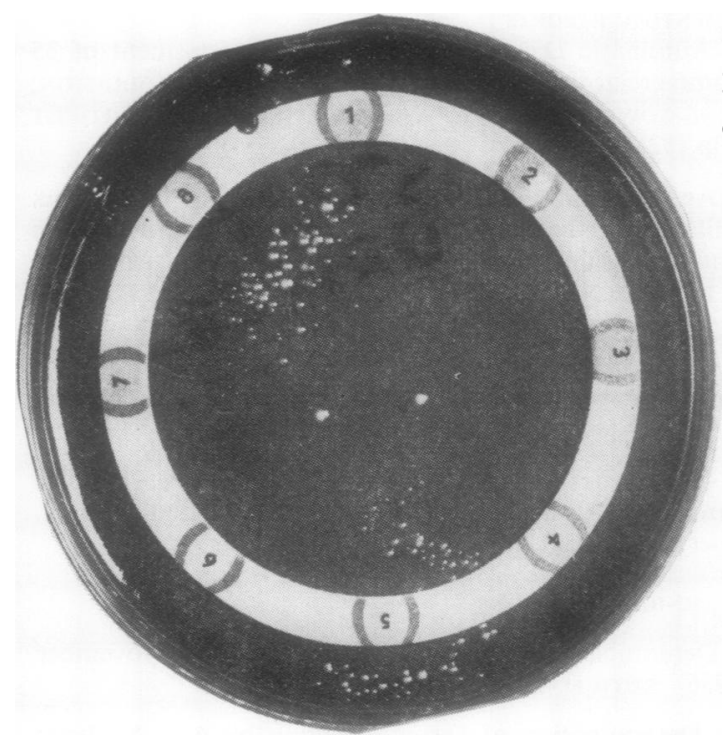

Fig. 1 Antibiotic sensitivity test of $\mathrm{Kl}$. aerogenes, DST lysed horse blood agar. Growth of the organism is limited to a small area centred about (8) sulphathiazole $(200 \mu \mathrm{g})$, and (5) nitrofurantoin $(200 \mu \mathrm{g})$ on the Mastring. The other antimicrobial agents are (1) ampicillin $25 \mu g(2) ;$ colistin methane sulphonate 200 $\mu g$; (3) kanamycin $30 \mu g$; (4) nalidixic acid $30 \mu g$; (6) streptomycin $25 \mu \mathrm{g}$; and (7) tetracycline $100 \mu \mathrm{g}$. 


\section{METHODS}

From an overnight broth or peptone water culture of the organism a $4 \mathrm{ml}$ tube of peptone water was inoculated with a straight wire. This was incubated at $37^{\circ} \mathrm{C}$ for about four hours until turbidity was just visible: a 1/1000 dilution of this culture was then made in sterile phosphate-buffered saline, $p \mathbf{H ~ 7 \cdot 3}$ (Dulbecco A Oxoid). The number of organisms in this suspension was of the order of $10^{4} / \mathrm{ml}$; variations between $10^{3}$ and $10^{5}$ organisms $/ \mathrm{ml}$ were permissible. The suspension was flooded onto the test plate, the excess fluid sucked off, and the plates were allowed to dry. The test plates had been previously matured by incubation at $37^{\circ} \mathrm{C}$ for four hours (Harper and Cawston, 1945). Appearances in the accompanying figures were not always easy to reproduce in so clearcut a way as shown. A light inoculum of a young culture gave the best results: older cultures produce aggregations of growth akin to pellicle formation which made them unsuitable for use, and if the inoculum was too heavy 'break through' growth took place, which, however, was usually light and easily distinguished from the stimulation effects to be described. Furthermore the inhibition of bacterial growth on different batches of horse-blood DST agar varied; some batches were less inhibitory than others, but we can suggest no reason for this.

Inhibitions between substances were tested using impregnated blotting paper strips applied to the medium at an angle of $90^{\circ}$ to each other. For titration of the extinction point, solutions were applied in porcelain insulator beads (fish-spine beads) and the zone diameters plotted in the usual way.

\section{Experimental Results}

Stimulation of growth was found with the sulphonamides (sulphamethoxazole and sulphanilamide), and substances chemically similar (Fig. 2) such as para-amino benzoic acid(PABA), para-amino salicylic acid (PAS), and procaine $\mathrm{HCl}$. Sulphanilamide and PABA were titrated decimally to extinction; sulphanilamide stimulation could be detected at a concentration of $10^{-5}$ molar, whereas PABA (or its more soluble sodium salt) stimulated only down to $10^{-2}$ molar concentration. In other words, as a growth stimulator, sulphanilamide was $10^{3}$ times more active than PABA mole for mole. Paraamino benzoic acid and procaine $\mathrm{HCl}$ interfered with the actions of sulphonamide and PAS (Fig. 2). Figure 2 also shows that PABA, at a concentration insufficient to stimulate growth, inhibited sulphonamide stimulation.

Two further effects were seen, which are illustrated in Figures 3 and 4. In Fig. 3 a sulpha-methoxazole

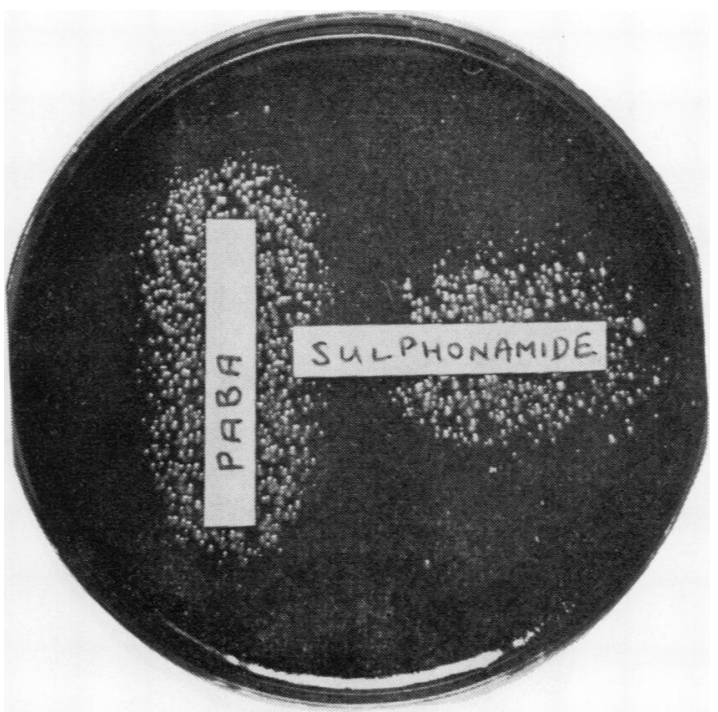

Fig. 2 Inhibition of sulphonamide activity by paraamino benzoic acid on DST lysed horse-blood agar seeded with Klebsiella. Blotting paper strips soaked in solutions of the two substances (sulphanilamide, M/1000; PABA, $M / 50)$ are surrounded by stimulated growth. Para-amino benzoic acid has, however, inhibited sulphonamide stimulation where present in a concentration too low to promote growth.

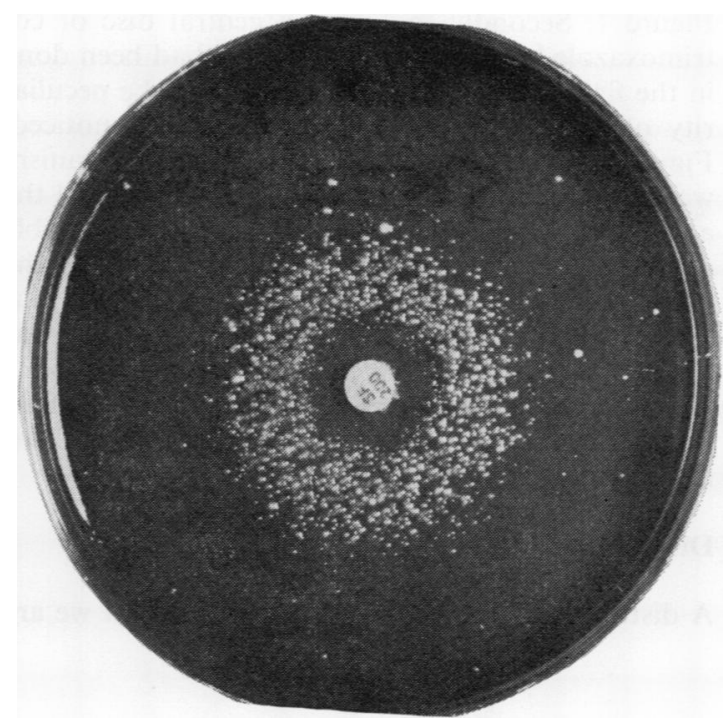

Fig. 3 Sensitivity plate seeded with Klebsiella. The central disc contains sulphamethoxazole $200 \mu \mathrm{g}$. Growth of the organism is first inhibited then stimulated by lower concentrations of the sulphonamide. 


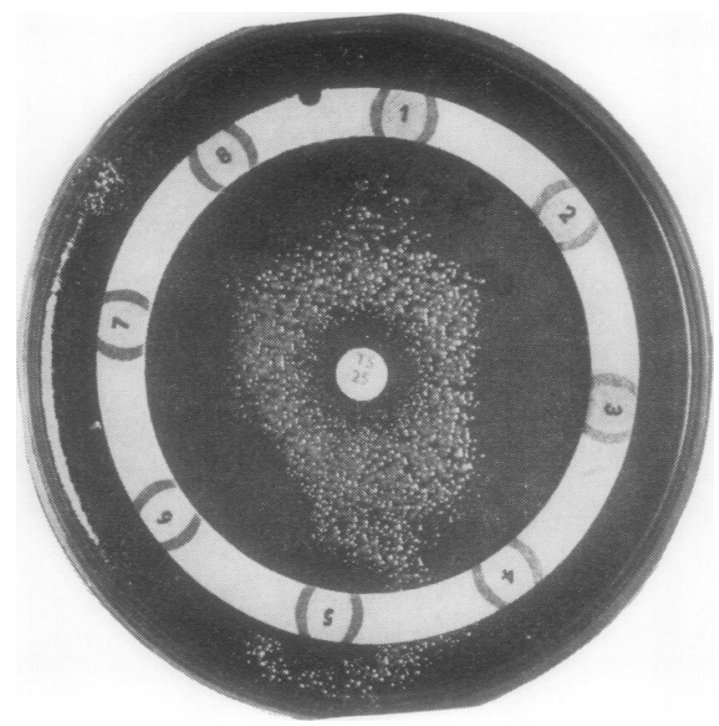

Fig. 4 Sensitivity plate identical to Fig. 1 but a disc impregnated with co-trimoxazole has been placed centrally. The effect of the chance application of this disc has been to stimulate growth around it and so apparently to make the test readable.

$200 \mu \mathrm{g}$ disc (Mast) has produced a zone of inhibition surrounded by a zone of stimulated growth; this is similar to but clearer than the effect shown in Figure 1. Secondly in Fig. 4 a central disc of cotrimoxazole has been applied; if this had been done in the first place, as is commonly done, the peculiarity of the organism would have gone unnoticed. Figure 4 could be read as indicating that the organism was sensitive to co-trimoxazole as well as to all the antibiotics on the annulus-with the possible exception of (1) ampicillin. However when the organism was tested on other media, DST agar without lysed blood, or Wellcotest agar, it was resistant to co-trimoxazole.

The growth-stimulating action of nitrofurantoin (Fig. 1) is weak but consistent. The reasons for this action have not been elucidated.

\section{Discussion}

A disturbing feature of this organism is that we are unable to judge from in-vitro tests whether it is or is not sensitive to co-trimoxazole or the sulphonamides. The latter appear to inhibit in high concentration and stimulate in low. By adjusting the amounts of thymine (or other substances) the organism may appear sensitive or resistant at will and the in-vitro tests cannot therefore be considered to be a useful guide to therapy.

Turning to the stimulating effect of sulphonamide, this seems to be a rather uncommon finding. We can discover references only to stimulation of the $\overrightarrow{0}$ Rickettsia of murine typhus (Snyder, Maier, and Anderson, 1942) and conversely some benefit to $\vec{\omega}$ clinical typhus ensuing on high dosage of PABA (Yeomans, Snyder, Murray, Zarafonetis, and Ecke, 1944). The present organism is, however, stimulated in vitro by both substances whilst the competitive inhibition between them remains unaltered. As the patient appeared not to have been treated with co-trimoxazole we do not know if his condition would or would not have been aggravated by giving these drugs, but the possibility of adverse effects should be borne in mind if similar organisms become common. Dr R. M. Maskell (personal communication) has also isolated co-trimoxazoleresistant, sulphonamide-stimulated Proteus mirabilis strains in Portsmouth so that the organism we are reporting is not unique.

Finally we think that this and similar organism may prove of use as a metabolic tool and of interest for work in vivo. It has certainly made us look very carefully at our routine procedures so that we do not miss, as we may have in the past, significant growth of coliforms and perhaps other organisms, which may exhibit peculiarities similar to those reported here.

\section{References}

Harper, G. J., and Cawston, W. C. (1945). The in-vitro determination of the sulphonamide sensitivity of bacteria. J. Path. Bact., 57, 59-66.

Snyder, J. C., Maier, J., and Anderson, C. R. (1942). Report to the Division of Medical Sciences National Research Council, December 26th. Quoted by Wooley, D. W. (1952). In A Study of Antimetabolites, p. 169. Wiley, New York.

Yeomans, A., Snyder, J. C., Murray, E. S., Zarafonetis, C. J. D., and Ecke, R. S. (1944). The therapeutic effect of para-aminobenzoic acid in louse born typhus fever. J. Amer. med. Ass., 126, 349 356. 